\title{
Consideraciones sobre las salas multiedad en el Nivel Inicial. Una investigación en jardines de Moreno
}

- Isabelino A. Siede, Cecilia Bernardi y Claudia V. Britez

\begin{abstract}
Resumen
Las salas multiedad son una forma alternativa de organización del Nivel Inicial con presencia cada vez mayor en la provincia de Buenos Aires. En contraste con la tradicional segmentación por edades, esta modalidad de agrupamiento suscita controversias y representaciones encontradas, al mismo tiempo que da lugar a nuevos ensayos y construcción de criterios para la enseñanza. En este artículo, se presentan los resultados preliminares de una investigación exploratoria sobre el funcionamiento de las salas multiedad en instituciones del partido de Moreno, orientada a indagar las representaciones de docentes, directivos y familias sobre ellas, los rasgos de la enseñanza que ofrecen y las condiciones institucionales que favorecen u obstaculizan su funcionamiento.
\end{abstract}

\section{Considerations about the multiage classrooms in Early Childhood Education. Investigation in kindergartens of Moreno district}

\footnotetext{
Abstract

The multiage classrooms are an alternative way of organization in Early Childhood Education, with increasing presence in Buenos Aires province. In contrast to the traditional age segmentation, this grouping modality raises controversies and conflicting representations. At the same time that it fosters new trials and building criteria for teaching. This article presents the preliminary results of an exploratory investigation on the working of multi-age classrooms in institutions of Moreno district.
}

\section{Palabras clave:}

Educación infantil, Nivel Inicial, sala multiedad, organización escolar.

Keywords:

Childhood education, Kindergarten, multiage classroom, school organization. 
1. Ambos proyectos se han desarrollado bajo la dirección del doctor Isabelino Siede y la codirección de la magíster Elisa Spakowsky. Como docentes investigadoras participan la profesora Claudia Brítez, la licenciada Mariana Vázquez, la licenciada Cecilia Bernardi y la profesora Romina

Petrolo. Como graduadas asistentes de investigación participan las icenciadas Patricia Arce, Nancy Agüero, Martha Zárate, Débora Spinosa y Paula Soledad López. Como becaria estudiantil participa la profesora Noelia Domini. Todo el equipo forma parte del Ciclo de Licenciatura en Educación Inicial de la Universidad Nacional de Moreno.

2. https://www.argentina.gob.ar/educacion/planeamiento/info-estadistica/ anuarios Fuente: Relevamiento Anual 2018, editado por la Dirección de Información y Estadística Educativa (DIEE) del Ministerio de Educación, Cultura, Ciencia y Tecnología.

3. Las secciones multinivel se comparten con el Jardín Maternal o el Nivel Primario.
Este artículo presenta un avance de los resultados del Proyecto de Investigación Científica y Desarrollo Tecnológico denominado "La enseñanza en las salas multiedad", presentado a la convocatoria PICYDT 2013 de la Universidad Nacional de Moreno y su continuación, el proyecto denominado "Comprensión crítica de la enseñanza en las salas multiedad del Nivel Inicial”, actualmente en curso. ${ }^{1}$

\section{Marco conceptual y presentación del problema}

La tradición dominante en la organización escolar del Nivel Inicial ha privilegiado la distribución de niñas y niños por salas de edades semejantes, lo cual lleva a que usualmente compartan las actividades cotidianas quienes tienen una diferencia máxima de doce meses. Se denomina "salas multiedad" a aquellas que reúnen niños y niñas de diferentes edades, es decir, de más de doce meses de distancia entre sí. En el Anuario Estadístico de Educación ${ }^{2}$ se reconoce a las primeras como secciones independientes y a las segundas como secciones múltiples.

Estas salas multiedad son muy numerosas en el sistema educativo argentino y en particular, en el sistema de la provincia de Buenos Aires. El relevamiento presentado en el Anuario Estadístico de Educación del 2018 indica que el segundo ciclo del Nivel Inicial, Jardín de Infantes, cuenta con 77694 secciones (tanto de gestión pública como privada) de las cuales 12650 corresponden a las denominadas salas multiedad. En la provincia de Buenos Aires se encuentran 5349, es decir, que representa el 42,28\% de todas las secciones multiedad de Jardín de Infantes del país. Y específicamente 2343 se hacen presentes en el Conurbano bonaerense comprendiendo así el 18,52\% de salas multiedad del país.

La preocupación que dio inicio a estos proyectos de investigación es que sobre ellas se ha construido una mirada estigmatizada, tanto desde la perspectiva de los equipos docentes como de la comunidad. En diferentes intercambios informales del sistema educativo bonaerense, las salas multiedad suelen ser definidas como "impuras", como secciones de "segunda categoría", en oposición a las llamadas "salas puras" donde concurren niños de una misma franja etaria. Por lo general, su apertura responde a cuestiones connotadas negativamente como la insuficiencia de matrícula o la escasez de aulas disponibles. En ese sentido, resulta interesante rescatar la pregunta acerca del potencial que la organización multiedad supone para la cobertura en el nivel planteada en el boletín n. ${ }^{\circ} 8$ de la Dirección Nacional de Información y Evaluación de la Calidad Educativa (DINIECE), Las cifras de la Educación Inicial y sus modelos de organización:

Se observa que, si bien las secciones independientes son en todos los casos mayoritarias, las múltiples y multinivel tienen una presencia que, en algunas jurisdicciones, representa una porción no desdeñable del total del nivel. Ante los problemas para aumentar la cobertura, cabe interrogarse si las secciones múltiples y multinivel, ${ }^{3}$ si se evalúan como pedagógicamente adecuadas, pueden considerarse una alternativa subutilizada para aumentar la matrícula con los recursos disponibles. (Dirié, 2010, p. 27)

A su vez, históricamente el Nivel Inicial estuvo atravesado por la tensión entre la función asistencialista y la función pedagógica (Feldman, 2009; Fernández País, 2018; Ponce, 2006) y es posible pensar que toda alternativa que se aleje del formato fundacional, en términos de la escuela primaria, sea considerada como un embate al reconocimiento del jardín como institución educativa. Sin embargo, diferentes autores y experiencias (Alpe \& Poirey, 2000; Feldman, 2009; Fu et al., 1999; Kinsey, 2002; Mugny \& Doise, 1978; Terigi, 2008) dan cuenta de la potencialidad educativa que supone la interacción de niños de diferentes edades en el marco de una propuesta didáctica. 
Las salas multiedad han sido escasamente estudiadas, generalmente desde programas gubernamentales y con expectativa de relevar experiencias valiosas para replicarlas en otros contextos (Coppa \& Saguier, 2007; Kuppe, Prudant \& Visintín, 2012; Laffranconi, 2011; Padawer \& Visintin, 2011; Serulnicoff, Massarini \& Negri, 2011). Según esos estudios, presentan en nuestro país diferentes características, según se ubiquen en zonas urbanas, rurales o de islas. El rango de edades; la cantidad de niños (salas de matrícula mínima); alumnos unidos por lazos de parentesco; la reiteración de la maestra, el aula y los materiales de un año a otro, son solo algunas de las variables que las determinan y que es necesario explorar con mayor profundidad. A partir de indagaciones realizadas tanto en el marco de experiencias comunitarias (Kaufmann, 2004) como en el sistema educativo (Serulnicoff, Massarini \& Negri, 2011) y el aporte bibliográfico, se evidencia que, pese a las particularidades que caracterizan a las secciones múltiples, no aparecen diferencias notorias con los modos habituales en los que se organiza la jornada de trabajo en toda sala de jardín. Es posible, tal como sostiene Flavia Terigi para la escuela primaria, que la ausencia de producción pedagógica y formación específica explique que los maestros extiendan -o busquen extender- a las aulas multiedad, los modelos didácticos del aula estándar (Ezpeleta, 1997; Terigi, 2008).

Desde el inicio del primer proyecto, hemos asumido la hipótesis por la cual estudiar estas secciones multiedad no solo aportaría producción de conocimiento sobre un área que resulta vacante, sino que, a la vez, la plurisala constituiría un analizador potente para examinar y revisar algunas de las variables duras que configuran la gramática escolar de la educación para la primera infancia como ser, la agrupación según la edad, la organización de la enseñanza según el supuesto de simultaneidad y de los aprendizajes en términos de monocronías. En épocas en que los discursos de la atención a la diversidad sobrecargan a las escuelas con demandas de difícil cumplimiento, la sección múltiple puede constituir un ámbito de indagación de posibilidades para la enseñanza en su conjunto (Terigi, 2008). Este tema resulta especialmente relevante en momentos de expansión del Nivel Inicial, dado que la obligatoriedad de la sala de cinco años se extendió recientemente a la sala de cuatro (Ley 27045, sancionada en diciembre de 2014), en consonancia con la Ley de Educación Nacional (26206, sancionada en diciembre de 2006). Asimismo, la Ley y el Plan Nacional de Educación Obligatoria (Resolución 188/12 del Consejo Federal de Cultura y Educación) habilitan y promueven la posibilidad de planificar y gestionar modelos organizacionales distintos al estándar, respondiendo a diversidad de contextos y adoptando múltiples criterios que trasciendan la variable edad.

Algunas de las preguntas que orientaron el primer tramo de esta investigación fueron las siguientes: ¿qué representaciones tienen docentes, educadoras comunitarias, directivos y familias sobre las salas multiedad? ¿Cómo se caracteriza el tratamiento de la enseñanza en las salas multiedad en jardines de infantes públicos de gestión estatal, privada o de las organizaciones comunitarias? ¿Qué condiciones institucionales favorecen $\mathrm{u}$ obstaculizan el funcionamiento de las salas multiedad?

\section{Rastreos preliminares y metodología de la investigación}

Durante los primeros años de trabajo conjunto, se procuró identificar las representaciones sociales de los equipos docentes, los directivos y coordinadores y las familias, sobre el sentido de las salas multiedad en jardines públicos de gestión estatal y en instituciones de las organizaciones comunitarias. Dentro de la investigación pedagógico-didáctica, se trata de un objetivo de carácter naturalista, pues intenta develar una trama representacional existente, abordado desde una metodología cualitativa. 
Mientras se definía la muestra de actores para entrevistar y se establecían los contactos institucionales, diferentes miembros del equipo se ocuparon de relevar estudios, documentos y datos que facilitaran el análisis posterior. Particularmente, se realizó un relevamiento cuantitativo de las salas multiedad del partido de Moreno, se compilaron algunas experiencias nacionales e internacionales valiosas y se exploraron referencias históricas a la organización de los jardines de infantes por salas homogéneas.

Entre los antecedentes históricos, se hallaron huellas de formatos organizacionales alternativos en las primeras experiencias de educación infantil en el país, mientras que luego se consolidó un modelo único basado en la homogeneidad etaria. Como ejemplo del primer caso, se puede citar el texto de la Baronesa Marenholtz-Bülow, que Sara Chamberlain de Eccleston tradujo para el estudio de sus alumnas, en la escuela normal de Paraná, durante la década de 1870 . Allí se describe una actividad usual en la propuesta de Froebel:

Un poco más lejos en el jardín, debajo de un toldo de lona, se ven tres mesas rodeadas de bancos con respaldos inclinados, y a cada una de las cuales están sentados diez niños, de cuatro a siete años de edad, que trabajan diligente y atentamente. Unos se ocupan en el tejido de tiras de papel, paja o cuero, en gran variedad de dibujos y de varios colores, para hacer carteras, cestillos, esterillas, cajitas, etcétera. Los dibujos de los niños mayores son de su propia inventiva, y sus obras están destinadas a regalos para sus padres y amigos. [...] Pero volvamos al jardín. Los niñitos que vimos ocupados en ejercicios gimnásticos, vienen ahora corriendo y riéndose a la mesa que dejaron los niños mayores, y toman sus asientos para una media hora de trabajo (a los niños más pequeños el tiempo de trabajo se les limita a un cuarto de hora) y principian a colocar y entrelazar pequeños listones o palitos en forma simétricas. "Formas de belleza" o construcciones sistemáticas sin objeto especial; "formas de conocimiento", o figuras matemáticas; "formas de la vida práctica" o útiles de labor, edificios, etcétera; o se dedican de algún otro modo a alguna de las numerosas ocupaciones cuyos resultados se ven expuestos en el armario de cristales colocado en la sala de recreo. (Von Marenholtz-Bülow, 1947, p. 112-114)

Como se puede apreciar, en esa descripción, no se menciona distinción de salas por edad, aunque sí hay actividades diferenciadas dentro de un grupo aparentemente heterogéneo. En aquella imagen de la educación infantil, no se planteaba una clasificación tajante por edades, sino que se articulaban momentos de trabajo conjunto entre niñas y niños mayores y menores, al tiempo que se establecían criterios de distinción como la limitación de tiempos.

Como contrapartida, un siglo después, el modelo de diferenciación por edades se había consolidado como ideal organizacional y así se lo presentaba en uno de los textos más profusamente utilizados en la formación docente:

Los niños de Jardín de Infantes, se agrupan por edades parejas formando secciones de 20 a 30 niños, bajo la conducción de una misma maestra. Estas cifras son las tolerables para que se mantengan las garantías de seguridad y buen manejo del grupo. Los niños que cohabiten en una sala deben ser de edades mentales parejas. Es una buena medida agruparlos por edades cronológicas (salas de 3, de 4, de 5 años) y luego separar aquellos que se destaquen por su inmadurez o por sus ventajas respecto al resto. La experiencia demuestra cómo se complica la tarea de la maestra que debe manejar grupos heterogéneos en que conviven niños de 2, 3, 4 y 5 años. Es imposible mantener la armonía en la satisfacción de intereses tan opuestos, 
es imposible focalizar la atención de niños con tan amplia curva de intensidad. ¿Cómo manejar el nivel de lengua ante los pocos recursos de los más pequeños y la avidez de los mayores? ¿Cómo seleccionar cuentos, poesías, unidades didácticas ante tal disparidad de intereses? ¿Cómo desdoblarse mientras unos duermen y los otros deben acudir al baño o realizar juego en los rincones? La ambición que lleva a admitir niños de toda edad en un mismo ambiente y con una misma maestra concluye por asfixiar en la docente toda ansia de trabajo positivo. Al comprobar sus impotencias para alcanzar ciertos logros, al ver destruidos sus esfuerzos y debilitada su voluntad por la imposibilidad del hacer efectivo se convertirá en una maestra que 'cuida niños', pero sin formar, sin orientar, realizando su tarea cotidiana sin la alegría propia del que construye. Las normas dictadas por la experiencia no son caprichosas sino sabias: los grupos deben ser homogéneos. Los grupos de tres años deberían contar con un máximo de 20 niños; los de cuatro con 25 y los de cinco con 30 , considerando siempre la presencia de dos adultos por grupo. Si, a pesar de las razones expuestas, se debieran agrupar niños de distinta edad, el promedio de sus edades determinará el número que alcanzará el grupo. No se desconocen las experiencias que se realizan actualmente en Inglaterra. Copiando el ya tradicional sistema Lancasteriano de la escuela primaria, se incluyen en los grupos de niños menores otros de mayor edad que servirán como guías, estimulando con sus posibilidades a los más pequeños. Pero en Inglaterra como en Estados Unidos de Norte América se trabaja con grupos reducidos de 15 a 18 niños en los que esta innovación, no asume los peligros que surgen en grupos de 30-45 niños. (Capizzano de Capalbo \& González Canda, 1976, p. 11-12)

Sin mayores detalles ni fundamentos teóricos, las autoras aluden a la experiencia como fuente de un criterio homogeneizador de las edades, condenando a la degradación de calidad y pérdida de la alegría a las alternativas que permitan diversidad de edades en un mismo grupo escolar. Niñas y niños habrían de ser agrupados primero por sus edades cronológicas y luego se reagruparía a quienes se reconociera con una "edad mental" diferente. Las autoras mencionan ciertas experiencias inglesas a las que parecen descalificar porque trabajan con grupos de edades diversas, pero reducidos en cantidad.

El contrapunto entre estos textos, invita a pensar que la intención de ser reconocido como nivel educativo llevó a los referentes del Nivel Inicial a cristalizar como deseable la forma organizacional más parecida a la que predominaba en la escuela primaria: grupos de edades homogéneas, bajo la tutela de un docente y con una propuesta formativa graduada (Baquero, Diker \& Frigerio, 2006; Caruso \& Dussel, 2003).

A partir de tales antecedentes, nos interesó indagar qué representaciones se expresan actualmente en torno a las salas multiedad del partido de Moreno, para lo cual construimos una muestra de escuelas a indagar. Según la caracterización de Stake (2010), nuestra investigación puede catalogarse como un estudio de casos instrumental ya que los casos se escogieron deliberadamente, como instrumentos para responder a los interrogantes planteados. El criterio más relevante para delimitar la muestra de instituciones escolares en la cual llevar a cabo el trabajo de campo fue que hubiera diversidad suficiente, siguiendo un parámetro de semejanza con la distribución de las instituciones en el partido de Moreno. En consecuencia, trabajamos con cuatro jardines de infantes de gestión estatal y dependencia provincial (tres de ellos con secciones multiedad y uno sin conformación de secciones multiedad) y un jardín comunitario. A fin de preservar la privacidad de las experiencias analizadas, denominamos a cada institución con una letra, según el siguiente esquema: 


\begin{tabular}{|l|l|l|}
\hline Jardín A & Comunitario & $\begin{array}{l}\text { Dos secciones multiedad. En turno mañana, sala de uno y dos años. En turno tarde, } \\
\text { sala de tres y cuatro años }\end{array}$ \\
\hline Jardín B & Gestión pública & $\begin{array}{l}\text { Sin salas multiedad, aunque cuenta con talleres multiedad en algunos tramos de } \\
\text { cada semana }\end{array}$ \\
\hline Jardín C & Gestión pública & Cuatro salas multiedad \\
\hline Jardín D & Gestión pública & $\begin{array}{l}\text { Una sala multiedad, en turno mañana, de cuatro y cinco años. También cuenta con } \\
\text { talleres multiedad }\end{array}$ \\
\hline Jardín E & Gestión pública & Dos salas multiedad \\
\hline
\end{tabular}

Dentro de cada institución, delimitamos los actores que podrían ofrecer miradas diferentes y complementarias. En consecuencia, entrevistamos a las directoras y realizamos grupos focales con docentes y con familias por separado. Se optó por la realización de entrevistas semiestructuradas, que se llevaron a cabo con un cuestionario guía enunciado como tópicos de diálogo, lo cual permitió la emergencia de nuevas categorías, ejerció un control mínimo sobre las respuestas de los informantes y promovió que ellos se expresaran en sus propios términos. De esta manera se intentaba comprender la perspectiva de cada persona, sin imponer las percepciones propias del investigador. Estas entrevistas cualitativas permitieron recoger información de la subjetividad de los actores, en aspectos como sentimientos, actitudes y motivaciones, pensamientos o ideas, significados atribuidos a cada término o categoría, recuerdos emblemáticos, etcétera.

El grupo focal, por su parte, es una técnica de recolección de datos que promueve la interacción del grupo sobre un asunto determinado por el investigador y, en este caso, permitió que el intercambio con colegas o con grupos familiares suscitara contrastes y detección de contradicciones entre los participantes. En este caso, cada grupo focal se centró en algunos tópicos prestablecidos de diálogo y la solicitud de ejemplos, en relación con ellos. Durante el segundo semestre de 2016, se llevaron a cabo catorce entrevistas y grupos focales con maestras, educadoras comunitarias, directoras, coordinadores y familias de instituciones de educación inicial de Moreno, desgrabadas luego en su totalidad.

Como herramientas específicas de análisis de la información recolectada, se utilizó el análisis crítico del discurso (ACD), que provee herramientas para relevar representaciones, valoraciones y justificación de prácticas, a través de lecturas textuales y contextuales que favorezcan una interpretación plausible en cada caso, al poner en relación cada enunciado con el discurso del resto de los sectores involucrados, con las resonancias históricas y con el contexto social:

El ACD es particularmente apropiado para el análisis crítico de políticas porque permite realizar un estudio detallado de la relación del lenguaje con otros procesos sociales y el modo en que el lenguaje opera dentro de las relaciones de poder. El ACD proporciona un marco para un análisis sistemático -los investigadores pueden ir más allá de la especulación y demostrar el modo en que efectivamente operan los textos de políticas-[...]. Es la combinación del análisis lingüístico con el análisis social lo que convierte al ACD en una herramienta particularmente útil para un análisis crítico de políticas en comparación con otros enfoques. (Taylor, 2009, p. 178)

Los tres relevamientos mencionados (relevamiento histórico de las ideas pedagógicas sobre distribución de salas de edades homogéneas en el Nivel Inicial, relevamiento de experiencias nacionales e internacionales sobre salas multiedad y relevamiento cuantitativo de las salas multiedad en el partido de Moreno, la provincia y el país) permitieron 
construir un marco referencial para el análisis de las representaciones expresadas en los grupos focales y entrevistas a familias, docentes y directivos de jardines de infantes de Moreno. En tal sentido, estas tareas ofrecieron un marco general de referencia para realizar una primera aproximación a las valoraciones de las salas multiedad en diferentes sectores del Nivel Inicial, en el partido de Moreno.

\section{Resultados y hallazgos}

Las entrevistas y grupos focales permitieron relevar una considerable variedad de aspectos, que permiten adentrarnos en las dinámicas institucionales y las representaciones que dan sustento a la tarea cotidiana de directivos y docentes en escuelas de Nivel Inicial del partido de Moreno. Aunque el curso de los intercambios no fue lineal y ordenado, es posible reconstruir a posteriori algunos tópicos que atraviesan la experiencia de todas las instituciones de la muestra. Los presentamos a continuación, junto con algunos fragmentos ejemplificadores.

Uno de los tópicos abordados, aunque en menor medida de lo esperado, fueron los factores que inciden para que las instituciones decidan adoptar un formato de sala multiedad. En varias instituciones, la experiencia se inició a partir de coyunturas particulares:

-[Se abrieron salas multiedad] por demanda de los nenes de cinco. Tenía que hacer ingresar a todos los del radio. Eran todos del radio y los excedentes que habían quedado del año pasado. [...] Había muchos excedentes y fue una forma de organizar para darle prioridad más a la sala de cuatro que empezó a ser obligatoria. Entonces, se les dio más espacio a los de cuatro y se empezaron las salas multiedad. (directora del Jardín C)

En varios casos, la existencia de salas multiedad se atribuye a deficiencias edilicias o, de forma implícita, a falencias del Estado para resolver una propuesta pedagógica adecuada. Es llamativo, en tal sentido, que se use habitualmente el adjetivo "pura" para referirse a las salas de edades homogéneas, connotando la "impureza" o calidad inferior de las salas multiedad:

-Vos venís a un colegio en el Estado. En el privado... Yo no sé si en los privados hay multiedad. Yo, al menos, en las que trabajé y conozco, son todas puras. No hay multiedad. Las que conozco. Capaz que hay... (maestra del Jardín C)

-Me parece que lo de multiedad es más que nada por falta de escuelas, falta de lugar, nada más. Si no, serían todas puras. (maestra del Jardín C)

En los testimonios hay escasas referencias a la posibilidad de proponer salas multiedad como alternativa innovadora que podría enriquecer la experiencia formativa de niñas y niños en la educación infantil:

-Yo había pensado en una [sala multiedad] de tres, cuatro y cinco, pero ahí ya no sé si las maestras no se iban a poner los pelos de punta. Por ahí es una buena estrategia. Veremos, a ver qué pasa. Es una posibilidad. A mí me encantaría pasar por esa experiencia, porque nunca tuve a cargo así y nunca, en los jardines que transité en tantos años, no había salas así: multiedad de tres, cuatro y cinco. Yo no sé si alguna maestra hubiese aceptado ese desafío. Primero, porque me encantan los desafíos, y segundo porque me parece una buena oportunidad para que ellos intercambien experiencias. Aparte, este jardín es un jardín que trabaja muy dentro de la sala. No son de trabajar así en talleres y de compartir. (directora del Jardín C) 
4. Veremos que algo semejante ocurre con la apreciación sobre las diferencias atribuidas a los rasgos diferenciales atribuidos a niñas y niños de edades distintas.
Asociado a la decisión de abrir salas con este tipo de configuración, hay variedad de reacciones y representaciones sobre las salas multiedad, que tomamos también como tópico de análisis y que reúne una variedad considerable de valoraciones.

Muchos de los docentes y directivos entrevistados aludieron a la resistencia de algunas familias ante la incorporación de los niños y las niñas que tienen a su cargo a este tipo de salas, que les resultaban raras o de menor calidad formativa:

-Cuando vos les decías "la sala multiedad”, no les gustaba mucho. “¿Qué es una sala?” "Pero el mío va a egresar ¿Por qué está en una sala...?” No entendían eso, por qué estaba con el de cuatro, como que se iba a quedar en el jardín viste. “¿Por qué, si le toca 5, está con el de cuatro?”. (docente del Jardín B)

-Primero, directamente "no me gusta que esté con los bebés" o, si no, la otra que te decían era que el hijo iba a hacer un retroceso. Bueno, así que había que explicarles, que hablarles, el por qué estaban los chiquitos". (coordinadora del Jardín A)

Algunas de las resistencias se originan en los equipos docentes y perduran aún durante el desarrollo de esta propuesta:

-Para mí, son mejores las salas puras. Igual, yo estoy justo en una sala multiedad cuatro y cinco, porque la elegí, pero lo mejor sería que fueran puras las salas. Te doy un ejemplo: yo tengo nenes que son egresados y otros nenes que no. Entonces tenés que trabajar con eso y a la familia también le cuesta. (maestra del Jardín C)

Sin embargo, en muchos casos se mencionan cambios en las valoraciones a partir de transitar la experiencia en las salas multiedad:

-Al principio cuesta. Al principio te preguntan: “¿Es de tres la sala?” “No, es de tres y cuatro” “¿No es de cuatro?” “No, es de tres y cuatro”. Después, durante el año, la verdad es que no hay quejas. Solamente una mamá que me pidió que el nene esté el año que viene en sala de cuatro, que no vuelva a estar en sala multiedad, pero es una cuestión de que ella tiene esa creencia de que por ahí ellos se atrasan, a pesar de que ve que el nene avanzó un montón [...]. Después, la mayoría no. Hay muchas mamás de sala de tres que pasan a sala de cuatro, que piden estar en sala multiedad. Es una elección también de ellas. (maestra del Jardín A)

En algunos testimonios se observa una minimización de los rasgos diferenciales entre las salas multiedad y las restantes o cierta ambivalencia entre el reconocimiento de las diferencias y su posterior negación. ${ }^{4}$ En general, esa caracterización se asienta en la semejanza con la crianza familiar, donde es frecuente que los adultos responsables tengan a su cargo niñas y niños de diferentes edades:

-Es como una madre, digamos, ¿no? Capaz hay muchas mamás que también tienen... Nosotros somos nueve hermanos: yo tengo mi hermana y mi hermano que nos llevamos un año los tres juntos. Un año justito. [...] Nosotros nos criamos juntos, digamos, porque es un año, nomás. No creo que para una maestra sea más trabajo un nene de cinco que un nene de cuatro. (mamá del Jardín B)

-Es un año que se llevan. Tampoco es mucho, ¿no? Está bien, porque igualmente hay algunos nenes que ya cumplieron cinco y él sigue teniendo cuatro. Así que es lo mismo. Mi sobrina va con él. Son primos ellos dos, y vienen juntos. Ella cumplió cinco ya... Creo que en mayo... sí, mayo. Y él todavía tiene cuatro. Hay muchos nenes que ya tienen los cinco y él cumple recién en febrero los cinco, así que, para mí, es lo mismo. Es un año, nomás. Tampoco es mucha diferencia... No me molestaría. (mamá del Jardín B) 
Un tercer tópico recurrente en los grupos focales y entrevistas es la alusión a las condiciones institucionales que favorecen u obstaculizan la organización de salas multie$\mathrm{dad}$, entre las cuales se mencionan los espacios, la infraestructura, el equipamiento y materiales, pero también algunas tradiciones institucionales y hábitos extendidos en los equipos docentes.

Los condicionantes materiales se mencionan en pocos casos y con soluciones bastante asequibles, tanto al referirse a la infraestructura edilicia como a la disponibilidad de materiales didácticos:

-El patio es lo único con lo que estamos desconformes. En realidad, el patio, como nosotras compartimos con la iglesia, tenemos todo un tema de no poder mejorar el patio. Entonces, bueno, nosotros salimos mucho afuera. Lo que hacemos es siempre reforzar el tema de la seguridad, pero salen afuera a pesar del patio. Pero después, con la sala, la verdad, la podés transformar como quieras, porque al ser tan grande está buenísimo. [...] Vos corrés todo, armamos peloteros, túneles, sacamos, ponemos lo que queramos. Los nenes juegan en el piso, en la mesa, en donde quieran. Entonces eso también está muy bueno. Todo el tiempo se cambian. Digamos, la sala, nunca está igual. Ni siquiera los muebles: cambiamos los muebles de lugar. (maestra del Jardín A)

-Los recursos cuestan mucho [elegirlos], porque hay cosas que capaz que le atraen más a los de cuatro y a los de cinco ya les parece aburrido. Como recién le estaba diciendo a una nena, que aparte parece más grande, parece de siete años. Le digo: “¿qué pasa Ama?” -“Es que me aburro con ellas”, me dijo. (maestra del Jardín C)

Los condicionantes subjetivos y las tradiciones vigentes en las instituciones parecen generar más rispideces y dificultad para afrontar el formato de las salas multiedad:

-El formato escolar es muy difícil de romper. Entonces, por más de que queremos ser innovadores, muchas veces, en algunas cosas mínimas, te das cuenta [de] que no. Y digo "pero hicimos esto para que los pibes sean más libres, puedan elegir". Cuando eligen, les decimos que tenían que hacer lo otro. (directora del Jardín D)

-Por el guardapolvo, porque a veces no es sala celeste, 5 como la de nosotros. Es rojo el guardapolvo y a ellas no les gusta. [...] Es otro color de guardapolvo, es diferente... Eso nomás, pero después... O sea, una, como mamá, quiere que el nene, en sala rosa, tenga el guardapolvo rosa, en sala celeste se usa el celeste y en sala verde, que es el último, verde. Acá hubo chicos egresados que fueron sala verde, y los de sala integral que tenían guardapolvo rojo... Eso nomás lo que diferencia. (mamá del Jardín B).

-No es solo su maestra, sino que es todo el jardín responsable de todos los nenes. Digamos que, por eso, cuando a veces no está la maestra, aunque no esté la suplente o está la preceptora o está la directora o tal vez ese día está jugando con otra sola, porque, digamos de alguna manera, todos somos responsables de que esté en el jardín. (maestra del Jardín D)

Entre esos condicionantes subjetivos, cobran particular protagonismo los equívocos y malentendidos en la relación con las familias:

-Y nos pasó que, por ejemplo, hay un nene que pasa de multiedad de tres y cuatro. Es de tres este año y pasa a la sala de cuatro. ¿Qué pasa? Como yo no tengo otra sala de cuatro continúa a la sala azul, que sería la multiedad, entonces a esas que son pocas, no son tantas, se les explicó a las mamás. “¿Cómo? Si ella ya hizo sala azul este año”. “Bueno, pero él es de tres. ¿Te acordás, mamá, que esa es sala de tres y cuatro?”. “Ah, sí, sí”. Bueno, no es que repitió, porque la mamá dice “¿cómo...?”. (directora del Jardín C)
5. En los Jardines de Infantes de Moreno, hay colores asignados para todas las salas de tres (rosa), salas de cuatro (celeste) y salas de cinco (verde). Tal asignación resulta habitual para docentes, directivos y familias de la zona y en buena parte de la provincia de Buenos Aires. La rigidez de esa distinción torna más visible la ex cepcionalidad de las salas multiedad. 
-A mí me pasó con esto de la articulación y con el campamento: “¿Por qué ellos van al campamento y nosotros no?” Vos les explicás a tu manera. Los padres también, porque les pedimos que les expliquen, pero, bueno, ellos tienen esa angustia todavía: por qué ellos tienen el distintivo y los otros no. [...] Una vez, una abuela me dijo: "ay, no digas delante de ella que van al campamento". Le digo: "no, ¿cómo que no? Si es la verdad. ¿Qué? ¿Le voy a mentir a la nena? Si es verdad que voy a ir al campamento". Aparte, ese día, ellos no iban a tener clase, los de cuatro. Estábamos todos afectados al campamento... Es un trabajo con los padres. En las reuniones se habló un montón de veces: "entiendan que el año que viene ellos van a ser los que van a ir al campamento, que van a hacer la articulación". (maestra del Jardín C)

-En la planificación, las chicas ya tenían organizado que tal día se iba a trabajar la articulación. Entonces se citaba o se les explicaba a los papás. Se les daba un horario distinto. [...] Al principio tenía que ver la dificultad con la organización: que el papá entendiera que no se lo reunía a él, que no era porque no estaba incluido, sino porque era algo que sus hijos lo iban a vivir el año siguiente. (directora del Jardín B)

En estos y otros testimonios, es posible advertir que tanto el color del guardapolvos como los rituales asociados al egreso del Nivel Inicial son vistos por los equipos docentes como algo inmodificable y que merecería mejor comunicación para que sea comprensible por parte de los grupos familiares, pero no admitirían soluciones alternativas, como quitar los colores preasignados a cada edad o llevar a niñas y niños a dos campamentos para no suscitar diferencias dentro de un grupo donde hay quienes egresan y quienes no. En estas cuestiones, pareciera haber problemas abiertos que podrían decantar en soluciones innovadoras con el transcurso de la experiencia en varias instituciones.

Uno de los tópicos más destacados y particularmente relevante para nuestros objetivos de indagación, era el referido a las posibilidades y riesgos en los aprendizajes sociales $y$ cognitivos que advierten los actores institucionales entrevistados.

En algunos testimonios se mencionan rasgos diferenciales entre niñas y niños de diferentes edades, según miradas que responden a la tradición institucional de división de salas y a los aportes de la psicología evolutiva en la formación de los docentes, pero muy fácilmente esos mismos rasgos son relativizados por la misma persona que los enuncia:

-En realidad, en algunas actividades se puede ver la diferencia entre ellos, pero eso tiene que ver también, capaz que... No sé. En una sala de tres pura capaz que también hay diferencias entre los chicos. Tiene que ver con las características de muchas cosas. (maestra del Jardín C)

-Los de dos están intentando hablar. Entonces, estamos ahí en esa etapa, pero... los de tres por ahí están explorando otras cosas. Son más inquietos. Sí, los de tres lo que tienen es eso ¿no? Más inquietos. Están explorando más todo el tiempo las cosas. Entonces ellos están más activos y los otros, los más chiquitos, los siguen o los miran y ya empiezan a querer hacer las cosas que hacen ellos. Por ahí, la diferencia sería esa: el tema de la exploración. Los de tres están como explorando más cosas; en cambio, los de dos es más de los juguetes y esas cosas, pero, igual, tampoco tanta diferencia. (maestra del Jardín A)

-Acá pasa que hay unas mellicitas que son de cinco años. Tienen un problema de salud y son muy chiquititas. Vos las ves y parecen de tres, pero son de cinco. Y los de cuatro que están en esa sala, que es la multiedad, la ayudan a ella que es de cinco. Entonces ahí te fijas que, a pesar de que son de cinco y las ven tan chiquitas, los demás 
las ayudan. Es como que el de cuatro, que tendría que ser al revés, el de cinco ayudar al de cuatro, pero mirá en este caso cómo las ven tan, digamos, con esta dificultad que los de cuatro las ayudan a ellas. (directora del Jardín C)

-Es exactamente como otra sala. Juegan, tienen más afinidad con este o no. No tiene nada que ver la edad acá. La edad no existe. (maestra del Jardín D)

-Creo que no hay que rotular nada, porque hay chicos que tienen cinco años y son muy bebotes en su juego, en su habla, y hay otros de cuatro que tienen más calle que vos y yo juntas. O sea que eso me parece que no. Me parece que eso lo hace uno como docente. (docente del Jardín B)

En algunos testimonios, las diferencias entre edades no se establecen en relación con el desarrollo individual, sino como respuesta a tareas o actividades propias de la sala. En tales casos, aparecen referencias a dificultades originadas en la diversidad etaria y también valoraciones positivas de la interacción entre niños y niñas de diferentes recorridos:

-Yo planteo la misma actividad para todos, pero las resoluciones son distintas o las maneras de trabajar son distintas, porque cada nene, de acuerdo a su edad... Bueno, muchas veces no, porque a veces no se nota que son... Creo que en realidad depende de cada uno de los nenes, cómo resuelven cómo trabajan. Se nota mucho, por ahí, en marzo la diferencia, pero después eso se... El que no puede, ayuda al otro, porque lo que tiene es que se ayudan unos con otros todo el tiempo. La verdad... muy bien se trabaja. (maestra del Jardín A)

-Hay que verlo como positivo y no negativo, que teniendo una sala multiedad, el de cinco apura al de tres y lo nutre, y no es el adulto ¿no? El juego hace, digo yo, jugar con uno de cinco, y yo, si estoy viendo la habilidad que tiene el de cinco, pierdo el interés por ahí en el de tres, y en el de cuatro. Yo digo: uno siempre pone al de tres y al de cinco porque es como los polos, la distancia. El de cuatro queda ahí. (directora del Jardín B)

-En el juego, por ahí los de cuatro no se enganchan con los de cinco. No les atrae lo mismo. El de cuatro se dispersa más rápido, que quizás el de cuatro ya está por cumplir los cinco, y el de tres está por cumplir los cuatro. Entonces hay como una variedad y yo encuentro por ahí resistencia. (maestra del Jardín C)

-Capaz que, en Educación Física, ¿viste?, son más dispersos y se dispersan más. También los de cinco están esperando tener Educación Física por los juegos. En cambio, los de cuatro es como que están más dispersos. O vienen y se quedan al lado mío y yo voy con ellos. Y cuando me vuelvo a ir a verlos, están de vuelta al lado mío pegados. (maestra del Jardín C)

-El de cinco es muy crítico en cuanto a que todo tiene que salir desde lo más realista posible y no le perdona a los más chiquitos cuando se equivocan, y viceversa, los más chiquitos son totalmente frescos y naturales. Entonces, vos planteás una improvisación y se enganchan todos y te salen cosas que te quedás... Y el de verde: "no, no, pará porque yo esto ya no lo hago porque soy grande". Entonces, encontrar la vuelta es un muy gran desafío para el docente. Poder encontrarte con cada uno en distintas edades, porque acá no estás trabajando con una sala de cuatro, ni una sala de tres, estás trabajando con tres edades distintas". (docente del Jardín B)

-Es un desafío, porque tenés nenes chiquitos. O sea, un año es un año, pero, bueno, me gusta. [...] Lo bueno es la relación, porque los nenes, más allá de las edades, se relacionan entre ellos. O sea, puedo ver la diferencia que capaz uno de cuatro tarde más, o no, en hacer una actividad, pero, por ejemplo, no sé, estamos pintando, bueno, 
obvio que el de cinco te hace un dibujo terrible, y el de cuatro bueno, tiene su tiempo. Y ellos saben que tienen que esperarlos. Los esperan. Eso me gusta, porque es también esto de los valores, que valoran también a sus compañeros, el saber esperar. No hay que apurar a los demás. (maestra del Jardín C)

- Los de cuatro eran muy chiquitos y a algunos les costaba quedarse, cuando empezamos con el proyecto de articulación. Entonces, los de cuatro se quedaban, íbamos con los de cinco y, por ahí, las maestras medio que... Se encuentra dificultad en eso y con el tema del cuaderno. Cuando empezaron con el proyecto del cuadernoagenda, ahí también se vio dificultado, porque ¿qué hacíamos con los de cuatro? (directora del Jardín C)

Otro tópico recurrente, que suscita comentarios unánimes, es la relación entre la tarea en sala multiedad y la formación de los docentes. En todos los casos, las personas entrevistadas mencionan que la preparación fue muy escasa o inexistente:

-En el profesorado, nada que ver: no se hablaba de la multiedad. [...] Fue todo un desafío, porque tener tres y cuatro... “¿Qué hago? ¿Para dónde voy? ¿Para los de tres o para los de cuatro?” Esas cosas que uno va viendo, ¿no? Cómo perfilo el grupo. Pero en el profesorado no, en aquella época. (docente del Jardín B)

-Primero estaría bueno que nos capaciten a los docentes, porque las capacitaciones que hay son buenas, pero falta, porque de esto no hay una capacitación. Al menos donde yo estudié nunca nos capacitaron para multiedad. (maestra del Jardín C)

-Yo no veo profesorados en los que se vea las salas multiedad. O sea, tenés los contenidos, los bajás de acuerdo a la sala, pero nunca te dicen: "bueno, tenés multiedad, se trabaja...". O sea, no hay herramientas en los profesorados. (Maestra del Jardín C)

Ante la ausencia de formación de base, el último tópico que nos interesa analizar es el que alude a los criterios y respuestas particulares que se van generando a partir del trabajo en las salas multiedad.

Muchas docentes y directoras hablan de sus propios aprendizajes al transitar una experiencia para la cual no habían sido formadas, apelando a sus intuiciones, sus cambios de posicionamientos y los intercambios entre colegas para buscar alternativas:

-En nuestra formación tampoco tuvimos eso de cuatro y cinco mezclados, ni de tres y de cuatro, porque era características de tres, de cuatro, y de cinco. Era así, todo dividido, todo por color, todo así ... Entonces, claro, al encontrarte con todo esto, decís “¿Qué hago? ¿Cómo lo manejo?” [...] Vos decís: "Bueno, voy a probar con la intuición, a ver qué me pasa. A ver, pruebo con este juego". [...] Si uno no se puede salir de esa estructura que tiene, que tengo que controlar... Porque también “¿Cómo lo hago? ¿Qué hago?" (docente del Jardín B)

-Por ahí no se dan cuenta lo que mueve trabajar así, este formato de multiedad, de correrme de una estructura de pensar esto es en tres, esto en cuatro, esto en cinco [...]. Siempre les digo que eso no hace que se olviden las características propias de la edad, porque si no se nos mezcla todo y pretendemos que todos logren lo mismo. Entonces, cuando tiene que ver con la adquisición de alguna destreza, alguna conducta, algún aprendizaje, no olvidarme ¿no? Yo digo: la actividad es igual para todos, la mirada en la evaluación no [...]. No [hay que] olvidarse las características propias de la edad, no olvidarse el grupo que ustedes tienen. (directora del Jardín B) 
-“Uy, tengo que planificar una actividad para el de tres, y una para el de cuatro y cinco". Lo que veíamos era una para el de tres y una para el de cuatro y cinco. Entonces con Susana siempre decíamos: "la actividad es la actividad, no es actividades diferenciadas, tiene que haber una misma propuesta”. Ahí surge bien el material de este año en todo lo que era la sala multiedad. (docente de Jardín B)

-Yo evalúo el proceso. El proceso desde que ellos comienzan hasta que ellos... Un proceso desde marzo hasta ahora. [...] No evalúo por edad. Evalúo individualmente los procesos que va haciendo cada nene. Así evalúo yo. Creo que todas evaluamos de la misma manera, respetando los tiempos de ellos, cómo ellos van resolviendo. Se evalúa desde ahí. (maestra del Jardín A)

- Creo que fuimos aprendiendo que había que mirar otras cosas, así como había que mirar que ya no eran las características de los nenes de tres, de cuatro y de cinco. Había que mirar a las infancias. Había que pensar en esto que no estaba tan atado a la mirada psicologicista, digamos, sino a un contexto que lo rodeaba a cada niño y a su realidad ¿no? social, comunitaria. (directora del Jardín D)

Como es esperable y, en algunos casos, muy valioso que suceda, la construcción de nuevas alternativas suscita algunas tensiones o malestares, que dan cuenta de un proceso en devenir:

-Por ejemplo, en el momento de usar el cuaderno-agenda, usaban el cuaderno los de cinco y los de cuatro usaban la hoja. (directora del Jardín C)

-Este año, la directora dijo que no, que quería nada más que sean los de sala de cinco, también fue un tema: “ipor qué ellos tienen cuadernos y nosotros no?” “Ustedes tienen la hoja”, les expliqué... Bueno, pero ellos quieren su cuaderno. [...] "Y me decían que tenía que poner, no sé, que les dé masa o que haga otra actividad con ellos aparte. Pero, a ver, si estoy con los de cinco, no puedo estar con los de cuatro, o que llame a la preceptora, pero también tenemos una sola preceptora, que también hay dos salas. [...] Mi opinión personal es que, en realidad, tendrían que tener todos los cuadernos. (maestra del Jardín C)

-Cuando yo tomé el cargo, las chicas me lo dijeron y lo habían planteado en la reunión: o sea, los de cinco van a trabajar como si fuesen de cinco. La sala integrada va a trabajar como los de la verde, que es una sala toda de cinco. No se van atrasar. Quizás yo, por ahí, veo, que a la integrada de cuatro y cinco le cuesta generar espacios como para... organizarse. Las salas puras, digamos, o verdes, es como que... Bueno, ya como que fluye todo. Las veo yo. A las chicas les cuesta organizarse. (directora del Jardín C)

El material compilado es bastante extenso y requiere de lecturas minuciosas y construcciones de categorías más finas de análisis, pero estos primeros hallazgos que presentamos nos permiten avanzar en la enunciación de algunas conclusiones parciales, centradas en los juicios de valor concernidos en las prácticas y decisiones de directivos y docentes o en la evaluación que realizan los grupos familiares de niñas y niños que concurren a salas multiedad.

\section{Conclusiones provisorias y perspectivas de continuidad}

En líneas generales, la indagación exploratoria realizada hasta el momento permite destacar que, más allá de las resistencias iniciales, en la mayor parte de las entrevistas 
y grupos focales se encuentra una imagen de las salas multiedad como una experiencia valiosa, potencialmente formativa y dúctil para explorar nuevas alternativas pedagógicas y didácticas. Reconocemos esa imagen a través de los siguientes indicadores:

》Valoraciones positivas de las propuestas de agrupamiento multiedad, resaltando el aporte para el aprendizaje y las relaciones sociales entre los niños.

"Comentarios positivos por parte de las familias, quienes destacan que sus hijos conocen a otros niños y adultos de la institución, y pueden desenvolverse así con mayor confianza y autonomía.

" Presencia de otras experiencias de agrupamiento multiedad en el conjunto de propuestas didácticas de las instituciones, como talleres, encuentros musicales, proyecto de cuidado de plantas.

Esa imagen positiva coexiste con algunos indicadores que dan cuenta de un devenir complejo y aún costoso para los equipos institucionales:

»Ciertas diferencias con la organización habitual de secciones por edad, vividas como dificultades por las docentes entrevistadas.

» Propuestas diferenciadas para los niños que egresan del nivel y que transitan su última sección en una sala multiedad.

» Algunas dificultades para comunicar y fundamentar la propuesta multiedad en el diálogo con las familias.

Varios de estos rasgos se distancian de las previsiones iniciales del proyecto, por lo cual conviene analizarlos con mayor minuciosidad. Se trata de conclusiones provisorias y sujetas a revisión, pero muestran, al mismo tiempo, algunas alternativas y nuevos cursos de acción para el equipo investigador, tanto como para las instituciones de la zona.

En líneas generales, si bien es claro que la formación docente no ha ofrecido herramientas pertinentes para el trabajo con salas multiedad a quienes tienen responsabilidad sobre ellas, también es notorio que los recorridos formativos y las tradiciones institucionales se presentan como los obstáculos más relevantes para apreciar virtudes y potencialidades de esta experiencia. Las anteojeras de la costumbre se ofrecen como base para asentar temores y desconfianza, mientras que la intuición y la exploración emprendida por varios de los equipos docentes entrevistados permiten considerar que las salas multiedad pueden ser algo más valioso que una respuesta circunstancial.

Asimismo, la experiencia de las salas multiedad da cuenta de una tensión irresuelta entre el intento de parecerse a la primaria -a fin de legitimar el carácter educativo del nivel inicial- en la búsqueda de modalidades de trabajo homogéneo o que buscan paliar las supuestas desventajas de la heterogeneidad en las salas multiedad y, a la vez, la preocupación por diferenciarse del formato escolar tradicional, valorando la multiedad como un formato alternativo. Quizás haya allí una pista para hacer inteligibles la ambigüedad de valoraciones sobre las salas multiedad, como algo que existe "porque no queda más remedio" o como un formato a promover.

Las preguntas que orientaron el primer tramo de este proceso investigativo apuntaron a caracterizar y ponderar las prácticas actuales y los desafíos inmediatos van en la misma dirección, pero pueden también ofrecer fundamentos para que, en una instancia ulterior, el equipo encare preguntas de carácter normativo o propositivo como las siguientes: ¿cómo conviene organizar y planificar la enseñanza en las salas multiedad? ¿Cómo resolver el trabajo con contenidos específicos vinculados con el próximo ingreso a la escuela primaria de los niños de cinco años, en el marco de 
estas salas? ¿Cómo promover las interacciones entre los niños de distintas edades y con diferentes niveles de dominio de los contenidos para favorecer, en todos ellos, nuevos aprendizajes? ¿Qué criterios de agrupamiento conviene tener en cuenta en el trabajo cotidiano? El abordaje de preguntas como esas supondría el pasaje a una investigación de carácter propositivo, lo cual conllevaría nuevos instrumentales de diseño y análisis de información. 


\section{- Referencias bibliográficas}

»Alpe, Y. \& Poirey, J. (2000). Modèles culturels des acteurs, stratégies scolaires et conditions de l'étude dans l'école rurale. En Troisième colloque international "Recherche et formation des enseignants". Didactique des disciplines et formation des enseignants: approche anthropologique. Marsella: IUFM de l'Académie d'Aix Marseille.

» Baquero, R., Diker, G. \& Frigerio, G. (comps.). (2006). Las formas de lo escolar. Buenos Aires: Del Estante.

"Capizzano de Capalbo, B. \& González Canda, M. L. (1976). Enciclopedia práctica pre-escolar: bases para la estructuración de un sistema educativo. Buenos Aires: Latina.

"Caruso, M. \& Dussel, I. (2003). La invención del aula. Una genealogía de las formas de enseñar. Buenos Aires: Santillana.

" Coppa, G. \& Saguier, A. (2007). La sala multiedad en la educación inicial: una propuesta de lecturas múltiples. Buenos Aires: Ministerio de Educación, Ciencia y Tecnología de la Nación.

» Dirié, C. (coord.). (2010). Temas de Educación. Las cifras de la Educación Inicial y sus modelos de organización, año 5 (8). DINIECE, Ministerio de Educación.

»Ezpeleta, J. (1997). Algunos desafíos para la gestión de escuela multigrados. Revista Iberoamericana de Educación, (15).

»Feldman, S. (2009). Escuela infantil y salas de juegos multiedad: del proyecto a la realización. Los Polvorines: Universidad Nacional de General Sarmiento.

» Fernández País, M. (2018). Historia y pedagogía de la educación inicial en la Argentina. Desde el proyecto sarmientino hasta los inicios del siglo XXI. Rosario: Homo Sapiens.

»Fu, D., Hartle, L., Lamme, L., Copenhaver, J., Adams, D., Harmon, C. \& Reneke, S. (1999). A Comfortable Start for Everyone: The First Week of School in Three Multi-Age (K-2) Classrooms. Early Childhood Education Journal, 27(2).

» Kaufmann, V. (coord.). (2004). Otra forma de ser maestros. Una experiencia de inclusión de docentes en Jardines Infantiles Comunitarios de la Ciudad de Buenos Aires. Relatos de Experiencias Pedagógicas 2004. Buenos Aires: Subsecretaría de Educación-Dirección General de Planeamiento Educativo, GCBA.

» Kinsey, S. (2002). La agrupación de edades múltiples y el logro académico. ERIC Digest. Julio 2002. EDO-PS-02-4. Universidad de Illinois.

» Kuppe, I., Prudant, E. \& Visintín, M. (2012). Las secciones multiedad de Nivel Inicial en el ámbito Rural. Tensiones y transformaciones de las Prácticas Educativas a partir del equipamiento de ludotecas escolares. Buenos Aires: PROMER, MECyT.

"Laffranconi, S. (coord.). (2011). Modelos organizacionales en la educación inicial. Buenos Aires: MECyT.

" Mugny, G. \& Doise, W. (1978). Factores sociológicos y psicosociológicos del desarrollo cognitivo. Anuario de Psicología, (18). Universidad de Ginebra.

"Padawer, A. \& Visintin, M. (2011). La organización de salas "multiedad" con itinerancia como propuesta de extensión del NI en la provincia de Entre Ríos. 
Tensiones en torno a la obligatoriedad y la universalización en contextos rurales. Buenos Aires: PROMER, MECyT.

"Ponce, R. (2006). Los debates de la Educación Inicial en le Argentina. Persistencias, transformaciones y resignificaciones a lo largo de la historia. En Malajovich, A. (comp.), Experiencias y reflexiones sobre la Educación Inicial. Una mirada latinoamericana. Buenos Aires: Siglo veintiuno Editores.

»Serulnicoff, A., Massarini, V. \& Negri, G. (2011). Las salas multiedad. Relato de una experiencia. En Portal ABC, Ideas para el aula, sitio web de Dirección General de Cultura y Educación de la provincia de Buenos Aires: http://abc. gov.ar/recursoseducativos/

» Stake, R. E. (2010). Investigación Cualitativa: El estudio de cómo funcionan las cosas. New York: The Guilford.

" Taylor, S. (2009). La investigación de políticas y cambios educativos en los "nuevos tiempos" mediante el Análisis Crítico del Discurso. En Pini, M. (comp.), Discurso y educación. Herramientas para el análisis crítico. Una introducción. Buenos Aires: UNSaM.

"Terigi, F. (2008). Organización de la Enseñanza en los Plurigrados de las Escuelas Rurales. [Tesis de Maestría]. FLACSO. Buenos Aires, Argentina.

»Von Marenholtz-Bülow, B. M. (1947). El niño y su naturaleza [Sara Chamberlain de Eccleston (trad.). Buenos Aires: Albatros.

\section{Isabelino A. Siede}

Doctor de la Universidad de Buenos Aires, orientación Ciencias de la Educación. Licenciado en Ciencias de la Educación (UBA). Docente e investigador de la Universidad Nacional de Moreno. Moreno, Argentina. Correo electrónico: siedeisabelino@gmail.com

\section{Cecilia Bernardi}

Licenciada en Ciencias de la Educación (UBA). Docente e investigadora en la Universidad Nacional de Moreno. Moreno, Argentina. Correo electrónico: ceciliabernardi@hotmail.com

\section{Claudia V. Britez}

Licenciada y profesora en Ciencias de la Educación (UBA). Docente e investigadora en la Universidad Nacional de Moreno e institutos de formación docente de la Ciudad Autónoma de Buenos Aires y provincia de Buenos Aires, Argentina. Correo electrónico: claudiabritez@gmail.com 
